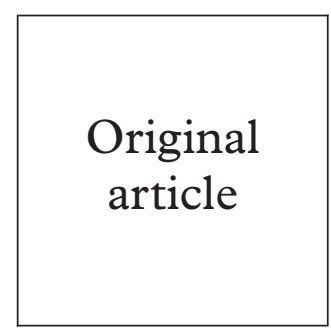

\title{
Factors associated with the time elapsed between the initial detection of HIV-1 antibodies and a diagnosis of AIDS among patients followed in Lyons University Hospitals
}

\author{
P Vanhems, D Baratin, R Allard, E Marceillac, F Biron, L Cotte, T Saint-Marc, and the \\ CISIH collaborators*
}

Objective: To identify the factors associated with a short period between the initial detection of HIV-1 antibodies and AIDS diagnosis among patients from Lyons, France.

Design and method: Prospective hospital based cohort study of patients diagnosed with AIDS in Lyons University Hospitals from 1994 to 1997. Cox regression was used to identify the variables independently associated with a short period between the first positive HIV-1 detection test and AIDS.

Results: 466 patients were studied, the mean period between the detection of HIV-1 antibodies and AIDS was 48 months and did not change across calendar years. Age $<46$ years (hazard ratio (HR) $0.77,95 \%$ confidence interval (CI) 0.58-1.00), HIV-1 transmission by heterosexual contact (HR 1.93, 95\% CI 1.49-2.51), Pneumocystis carinii pneumonia (HR 1.67, 95\% CI 1.28-2.17), or Kaposi's sarcoma (HR 1.42, 95\% CI 1.06-1.90) as the first AIDS defining event, and CD $4+$ count $<100 \times 10^{3} / \mathrm{ml}$ (HR $1.25,95 \%$ CI $\left.1.02-1.55\right)$ were associated with a short time interval between detection of HIV-1 antibodies and AIDS.

Conclusion: Educational interventions focused on heterosexuals and those aged over 45 are needed to promote the early detection of HIV infection, in the hope of reducing transmission and improving individual prognosis.

(Sex Transm Inf 1999;75:389-391)

Keywords: HIV infection; AIDS diagnosis

\section{Department of \\ Epidemiology and Public Health, Claude Bernard University, Lyon, France \\ $P$ Vanhems}

University Institute for International Health

(Lyon Centre)

$P$ Vanhems

\section{CISIH, Lyon}

University Hospitals,

Lyon, France

D Baratin

E Marceillac

F Biron

L Cotte

T Saint-Marc

Regional Health Board and Department of

Epidemiology and

Biostatistics, McGill

University, Montreal,

Canada

R Allard

$\star$ The collaborators are listed at the end of the paper.

Correspondence to:

Dr Philippe Vanhems,

Laboratoire d'Epidémiologie

et de Santé Publique,

Université Claude Bernard

Lyon 1, 8, av Rockefeller,

69373 Lyon Cedex 08,

France

Accepted for publication

22 September 1999

\section{Introduction}

The diagnosis of HIV-1 infection at the same time as or a few weeks before AIDS is not rare. ${ }^{1}$ This late linkage with medical care decreases the benefit of receiving antiretroviral therapy and prophylaxis against opportunistic complications. ${ }^{2}{ }^{3}$ From the public health point of view, these individuals could have transmitted the virus to others, as they did not know that they were infected. Age, sex, date of infection, heterosexual route of infection, geographic location, and being non-white were associated with a long interval between HIV-1 detection and AIDS. ${ }^{4}$ Samet et al reported that history of injection drug use, not having a living mother, not having a spouse or partner, not being aware of HIV-1 risk before testing, and being notified of HIV status by mail or telephone were associated with a longer interval from testing HIV-1 positive to presentation for primary care. ${ }^{5}$ The objective of our study was to identify the factors associated with the time between the first positive HIV screening test and an AIDS hospital diagnosis in patients from Lyons, France.

\section{Method}

PATIENTS

All patients who underwent for AIDS diagnosis at the Lyons University Hospitals based on the European AIDS definition ${ }^{6}$ between 1 January 1994 and 30 September 1997 were included. These patients were enrolled in a ongoing anonymous hospital based AIDS surveillance. ${ }^{7}$ At the patient's first visit for AIDS the following data were collected: age, sex, presumed route of HIV infection, date of the first HIV-1 positive screening test, CD $4+$ cell count $\left(\times 10^{3}\right)$ $\mathrm{ml}$ ), calendar year of AIDS, and AIDS diagnostic event. The date of the first HIV-1 positive screening test was based on the patient recall or on the test results if available. The HIV-1 infection was confirmed at AIDS diagnosis by western blot assay for all patients.

DATA ANALYSIS

The $\chi^{2}$ test and one way analysis of variance (ANOVA) were used for comparisons. A Cox regression analysis was performed with the time between the first HIV-1 positive screening test and AIDS diagnosed at the hospital as the dependent variable (or survival time). The association of variables with a short interval between first detection of HIV-1 antibodies and AIDS was quantified by adjusted hazard ratios with their 95\% confidence intervals (95\% CI). The following interactions were tested (sex by CD4+ cell count, sex by route of infection, route of infection by age and age by CD4+ cell count, year by CD4+ cell count and year by age). A two tailed $p$ value of $<0.05$ was considered significant.

\section{Results}

A total of 466 patients were analysed and described in table 1 . We did not find differences 
Table 1. Characteristics of 466 patients diagnosed with AIDS in Lyons University Hospitals between 1 fanuary 1994 and 30 September 1997

\begin{tabular}{ll}
\hline Characteristics & No(\%) \\
\hline Sex & \\
Female & $61(13)$ \\
Male & $405(87)$ \\
Age (years) & \\
$18-32$ & $126(27)$ \\
$33-37$ & $109(23)$ \\
$38-46$ & $116(25)$ \\
$\geqslant 47$ & $115(25)$ \\
Presumed route of HIV-1 infection & \\
Homosexual contact & $231(50)$ \\
Heterosexual contact & $132(28)$ \\
Injecting drug use & $50(11)$ \\
Other & $53(11)$ \\
Year of AIDS diagnosis & \\
1994 & $75(16)$ \\
1995 & $163(35)$ \\
1996 & $160(34)$ \\
1997 & $68(15)$ \\
Living in the Lyons area & $319(68)$ \\
CD4+ $\times 10^{3} /$ ml at inclusion $(\%)$ & \\
$0-50$ & $220(47)$ \\
$51-100$ & $81(17)$ \\
$101-200$ & $89(19)$ \\
$201-350$ & $57(12)$ \\
$>350$ & $19(5)$ \\
CD $4+<100 \times 10^{3} /$ ml & $301(64)$ \\
AIDS diagnoses & \\
Pneumocystis carinii pneumonia & $110(24)$ \\
Oesophageal candidiasis & $70(15)$ \\
Kaposi's sarcoma & $87(19)$ \\
Cytomegalovirus infection & $40(9)$ \\
Toxoplasma encephalitis & $23(5)$ \\
Other diagnoses & $136(30)$ \\
\hline
\end{tabular}

* Route of HIV-1 infection unknown $(n=42)$, transfusion recipient $(n=6)$, homosexual and injecting drug routes of HIV-1 infection $(n=5)$.

in proportion for sex $(p=0.78)$, age $(p=0.38)$, and route of infection $(\mathrm{p}=0.33)$ across calendar year but a statistical linear trend was detected concerning older patients $(\geqslant 47$ years old) in recent years ( $\chi^{2}$ for trend 5.36, $1 \mathrm{df}, \mathrm{p}=0.021$ ). The interval between the first positive HIV test and AIDS was on average 48 (SD 42) months and did not differ by calendar year $(p=0.93)$. Table 2 shows the variables independently associated with a short interval between the

Table 2 Variables independently associated with a diagnosis of AIDS being made soon after a first detection of $H I V-1$ antibodies

\begin{tabular}{|c|c|c|c|}
\hline Variable & $\begin{array}{l}\text { Hazard } \\
\text { ratio }\end{array}$ & $95 \% C I$ & $p$ Value * \\
\hline Age (years) & & & 0.0007 \\
\hline $16-32$ (reference stratum) & 1.00 & - & \\
\hline $33-37$ & $0.76+$ & $0.58-0.99$ & \\
\hline $38-46$ & $0.77 \ddagger$ & $0.59-1.00$ & \\
\hline$\geqslant 47$ & 1.27 & $0.97-1.66$ & \\
\hline Male sex & 1.27 & $0.93-1.75$ & 0.12 \\
\hline Presumed route of HIV infection & & & $<0.0001$ \\
\hline Homosexual contact (reference stratum) & 1.00 & - & \\
\hline Injecting drug use & 0.93 & $0.66-1.30$ & \\
\hline Heterosexual contact & $1.93^{\star \star}$ & $1.49-2.51$ & \\
\hline Other & 1.35 & $0.90-1.85$ & \\
\hline Leaving in Lyons area & 0.97 & $0.79-1.19$ & 0.77 \\
\hline Year of AIDS diagnosis & & & 0.17 \\
\hline 1994 (reference stratum) & 1.00 & - & \\
\hline 1995 & 0.92 & $0.69-1.21$ & \\
\hline 1996 & 0.77 & $0.58-1.02$ & \\
\hline 1997 & 0.75 & $0.52-1.06$ & \\
\hline $\mathrm{CD} 4+<100 \times 10^{3} / \mathrm{ml}$ & 1.25 & $1.02-1.55$ & 0.03 \\
\hline AIDS defining event & & & $<0.0001$ \\
\hline Other diagnosis (reference stratum) & 1.00 & - & \\
\hline Pneumocystis carinii pneumonia & $1.67^{\star \star}$ & $1.28-2.17$ & \\
\hline Oesophageal candidiasis & 0.77 & $0.57-1.05$ & \\
\hline Kaposi's sarcoma & 1.425 & $1.06-1.90$ & \\
\hline Cytomegalovirus infection & 1.17 & $0.81-1.70$ & \\
\hline Toxoplasma encephalitis & $0.59+$ & $0.37-0.95$ & \\
\hline
\end{tabular}

${ }^{\star}$ Global $\mathrm{p}$ value for the categorical variables.

$t \mathrm{p}<0.05, \neq \mathrm{p}=0.05, \mathbb{\mathrm { p }}<0.01,{ }^{\star \star} \mathrm{p}<0.001$

Model characteristics for the Cox regression: -2 Likelihood: 4786.790, $\chi^{2}: 92.546,17 \mathrm{df}$, $\mathrm{p}<0.0001$. first positive HIV test and an AIDS diagnosis. We observed a trend between age strata with a protective effect before 46 years of age and a higher risk after, without reaching the statistical significance level $(p=0.09)$. Other risk factors were heterosexual route of HIV infection, CD4+ cell count, and Pneumocystis carinii pneumonia or Kaposi's sarcoma as AIDS defining events. No interaction tested in the model was statistically significant ( $p$ values ranged from 0.86 to 0.15 ).

\section{Discussion}

The objective of this study was to identify the characteristics of patients who have had their diagnosis of HIV-1 infection shortly before AIDS. This suggests that HIV infection diagnosis has been done at a stage of advanced immunosuppression and is confirmed by a CD $4+$ count $<100 \times 10^{3} / \mathrm{ml}$ associated with a short time between the first positive HIV test and AIDS.

This detection delay between HIV infection and AIDS was stable between 1994 and 1997. However, use of highly antiretroviral therapy (HAART) could modify this delay in the future but that effect was not yet detectable.

Characteristics of patients with AIDS between 1994 and 1997 remain stable, but some trends need to be highlighted. Firstly, the proportion of AIDS patients infected by heterosexual contact increased from $24 \%$ in 1994 to $38 \%$ in 1997, while the proportion of men who had sex with men and injecting drug users decreased from $52 \%$ and $12 \%$ to $45 \%$ and $5.9 \%$ respectively during the same period. However, these trends were not statistically significant ( $p=0.14, \chi^{2}$ for trend). Secondly, we observed that those aged over 46 years increased from $21.3 \%$ in 1994 to $35 \%$ in 1997 $(\mathrm{p}=0.021)$.

This study conducted in Lyons is similar to the investigation performed by Porter et al in 1992. ${ }^{4}$ They found that heterosexual transmission and age over 50 was associated with being unaware of HIV infection less than 9 months before AIDS and this result is still valid in 1997 according to our data. We analysed the data according to a time interval of 9 months and we found that age $\geqslant 47$ years old, the year 1997, heterosexual route of infection, a PCP diagnosis, male sex and $\mathrm{CD} 4+<100 \times 10^{3} / \mathrm{ml}$ were associated with a time $<9$ months between the first positive HIV test and AIDS. This finding raises the possibility of a lack of impact of health education messages among heterosexuals at risk of HIV-1 infection and individuals aged in their late 40s. The absence of effect of not living in the Lyons area is reassuring, because it means that living far from an university medical centre was not associated with a higher risk of being seen later in the course of infection.

PCP remained the most frequent AIDS diagnosis, as elsewhere, ${ }^{9}$ and was strongly associated with a short time between HIV-1 infection detection and AIDS. This result suggests that a short interval between detection of HIV infection and AIDS did not provide the opportunity to prevent PCP; but it could be also that 
the HIV testing was prompted by early symptoms of PCP. On the other hand, for patients with an earlier detection of HIV infection, a lower incidence of PCP as an AIDS defining event corroborates the results of clinical trials showing the efficacy of primary PCP prophylaxis combined with antiretroviral therapy. ${ }^{23}$ Kaposi's sarcoma was also associated with a short delay between HIV detection and AIDS. That result is expected while patients with lesions suggesting Kaposi's sarcoma require a diagnosis of HIV infection.

We must address some limits of this study. The recruitment can be biased by sicker patients attracted to a university centre. We do not have the data to estimate if sicker patients were attracted in our centre over time or if certain risk group prefer the university centre. The date of the first HIV-1 positive screening test cannot be confirmed for all patients; however we think that this information was important enough in the individuals' life that the memory bias is limited.

In summary, age and a heterosexual route of HIV infection were associated with a late HIV-1 infection detection. Those individuals who had their diagnosis of AIDS shortly after the first positive HIV test highlight the need for innovative means of health message communication and for medical care strategies to slow the epidemic and improve individual prognosis.

Contributors: PV performed statistical analyses and wrote the Contributors. PV performed statistical analyses and wrote the paper; tion, and participated in writing the paper; RA contributed
. towards analysis, writing of the paper, and critical appraisal; EM contributed to the data collection and critical appraisal; FB, LC and TS-M are clinical referent physicians for this study and contributed to access to the data, to the result interpretation, and provided the clinical input of the paper.

The University Institute for International Health (Lyon Cenre) is a partnership between the University of Geneva (Switzerland), the University of Turino (Italy), the Fondation Merieux (Lyon, France) and the University Claude Bernard Lyon1
(France).

The authors are indebted to R-P Gilibert for the data manag ment and to the following collaborators who provided or gave access to the data: F Bailly, P Barlet, F Bissuel, A Boibieux, J-P Bonnin, D Bouhour, F Brunel-Dalmas, E Carbonnel, P Chiarelo, C Chidiac, P Chossegros, A Claudy, D Peyramond, M Faure, V Gueripel, F Jeanblanc, J-J Jourdan, J-M Livrozet, D Makloufi, P Miailhes, C Pariset, C Trépo, S Radenne, G Retornaz, P Rougier, I Schlienger, L Thomas, J-L Touraine, and J Fabry.

1 Poznansky MC, Coker R, Skinner C, et al. HIV positive patients first presenting with an AIDS defining illness: characteristics and survival. BMF,1995;311:156-8.

2 Volberding PA, Deeks SG. Antiretroviral therapy for HIV infection. FAMA 1998;279:1343-4.

3 Hirschel B, Lazzarin A, Choppard P, et al. A controlled study of inhaled pentamidine for primary prevention of Pneumocystis carinii pneumonia. N Engl f Med 1991;324: 1079-83.

4 Porter K, Wall PG, Evans BG. Factors associated with lack of awarness of HIV infection before diagnosis of AIDS BMF 1993;307:20-3.

5 Samet JH, Freedberg KA, Stein MD, et al. Trillion virion delay: time from testing positive for HIV to presentation for primary care. Arch Intern Med 1998;158:734-40.

6 Révision de la définition du SIDA en France. Bull Epid Hebd $1993 ; \mathbf{1 1}: 47-8$.

7 Vanhems P, Baratin D, Trépo C, et al. The recent dramatic decrease in AIDS cases, and HIV-related deaths contrasts with stable rate of new HIV-positive patients detected in with stable rate of new HIV-positive patients detected in
Lyon, France, from 1988 to 1997. F Acquir Immune Defic Syndr Hum Retrovirol 1999;20:316-7.

8 Phillips AN, Lazzarin A, Gonzales-Lahos J, et al. Factors associated with the CD4+ lymphocyte count at diagnosis of acquired immunodeficiency syndrome. The AIDS in Europe Study Group. 7 Clin Epidemiol 1996;49:1253-8.

9 Pezzotti P, Napoli PA, Acciai S, et al. Increasing survival time after AIDS in Italy: the role of new combination antiretroviral therapies. AIDS 1999;13:249-55. 